\title{
Urolithiasis is associated with low serum testosterone levels in men
}

\author{
Alper Otunctemur ${ }^{1}$, Emin Ozbek ${ }^{1}$, Suleyman Sami Cakir ${ }^{2}$, Murat Dursun ${ }^{3}$, Emre Can Polat ${ }^{4}$, \\ Levent Ozcan ${ }^{5}$, Huseyin Besiroglu ${ }^{1}$ \\ ${ }^{1}$ Okmeydani Training and Research Hospital, Department of Urology, Istanbul, Turkey; \\ ${ }^{2}$ Bayburt State Hospital, Department of Urology, Bayburt, Turkey; \\ ${ }^{3}$ Bahcelievler State Hospital, Department of Urology, Istanbul, Turkey; \\ ${ }^{4}$ Istanbul Medipol University, Faculty of Medicine, Department of Urology, Istanbul, Turkey; \\ ${ }^{5}$ Derince Training and Research Hospital, Department of Urology, Kocaeli, Turkey.
}

\begin{abstract}
Summary Objective: To evaluate the relationship among urolithiasis, metabolic syndrome (MetS) and serum testosterone (T) level in men.

Material and Methods: 513 men older than 18 years were enrolled in this study: 313 of the subjects had a history of stones (group 1) and 200 had no history of stones (controls, group 2). Early morning $T$ levels were recorded and anthropometric measurements were invastigated to evaluate MetS. Analyses were completed using chi-square tests. Result: Serum T level was lower in stone forming patients than coltrol subjects and $161(\% 51.4)$ men in group 1 and 92 (\%46) men in group 2 were diagnosed with metabolic syndrome. $T$ level was found lower limit $(<285 \mathrm{ng} / \mathrm{dl})$ in the MetS and urolithiasis group (p 0.002, OR 2.71). Conclusions: We found low testosterone levels in the patients with stone disease and prevalance of the MetS in men with urolithiasis was higher than in men without stone disease. Our findings show that levels of testosterone had no effect on stone formation, but the factors that cause stone formation can have an effect on the level of testosterone.
\end{abstract}

KEY WORDS: Urolithiasis; Metabolic syndrome; Testosterone.

Submitted 29 September 2014; Accepted 30 November 2014

\section{INTRODUCTION}

Metabolic syndrome (MetS) is an important public health problem worldwide, and its prevalence is increasing (1). MetS, the simultaneous occurrence of hyperglycemia, hyperlipidemia, hypertension, and visceral obesity, is a chronic disease associated with high mortality. In addition, this condition substantially increases the risk of developing cardiovascular diseases and type 2 diabetes (2).

Throughout the years, numerous definitions of MetS have been proposed by various organizations. Each of the definitions shares many similarities, including the presence of criteria relating to obesity, hyperglycemia, dyslipidemia, and hypertension (HTN).

However, several differences among the classifications are noted. The National Cholesterol Education Program Adult Treatment Panel III (ATP III) definition is the one most used today because it incorporates the key concepts of MetS; it relies on commonly used laboratory studies available to most physicians, and is less restrictive than the other classifications (3).

MetS has been linked to a number of urologic diseases including nephrolithiasis, benign prostatic hyperplasia and lower urinary tract symptoms, erectile dysfunction, male infertility, female incontinence, and prostate cancer (1). The prevalence of urolithiasis ranges from 2 to $20 \%$ throughout the world based on the geographic and socioeconomic characteristics of the different populations. The worldwide prevalence of the disease appears to have increased in the last quarter of the twentieth century for both men and women. The identification of common, modifiable risk factors for kidney stones may result in new approaches to the treatment and prevention of urolithiasis (4). Much like MetS and obesity, the prevalence of nephrolithiasis in the United States and other countries is increasing. There is evidence that these parallel changes might be linked (5). Studies have shown that MetS and its components (obesity/increased waist circumference, HTN, etc.) are associated with increased rates of nephrolithiasis (6-8). Although the exact pathophysiologic mechanisms underlying the association between metabolic syndrome and nephrolithiasis are unclear, hovewer, metabolic syndrome has been associated with changes in urinary constituents, including lower urinary $\mathrm{pH}$, decreased citrate excretion, and increased uric acid and calcium excretion, leading to increased risks of uric acid and calcium stone formation (9-12).

There is growing evidence that testosterone in men plays a major role not only in aspects of sexual health, but also in muscle and bone mass maintenance, erythropoiesis, and even glucose and lipid metabolism. Low testosterone levels are significantly associated with prevalence of MetS $(13,14)$. It is well established that urolithiasis occurs with greater frequency in males. The relative frequency has been noted to be three times that of women in multiple studies, and the rate of formation of idiopathic calcium 
stones has been reported to be four to five times higher in men than in women (15-19). This sexual disparity seems to be influenced by age. Interestingly, stone formation in the prepubescent population is similar between males and females (20). The greatest difference in idiopathic calcium oxalate stone formation is seen in the third and fourth decade of life (21). In the sixth decade of life, stone incidence, as well as testosterone levels, begin to decline in men (22-24). Each of these observations supports a role for sex hormones in lithogenesis. We aimed to invastigated relationship among serum testosterone levels, metabolic syndrome and urinary stone disease in this study.

\section{Materials AND Methods}

Between January 2008 and February 2012, we prospectively invastigated biochemical parameters and anthropometric measurements (height, weight, and waist circumference) of 313 men as the study group (Group 1) who visited our urology clinic for urinary tract stone and of 200 randomly selected men who had no history of stone as controls (Group 2).

Patients who had experienced spontaneous stone passage, had a surgery for urolithiasis (percutaneous nephrolithotomy, ureterorenoscopy) or whose stones were radiologically (ultrasonography, computed tomography or intravenous urography) visible at the time of clinical symptoms, were enrolled the study. Patients were excluded from the study if they had primary hyperparathyroidism, chronic diarrheal syndromes, intestinal malabsorption, complete distal renal tubular acidosis, primary hyperoxaluria, recurrent or active urinary tract infection, history of kidney transplantation, ongoing 5-alpha reductase inhibitor therapy, liver disease, primary gout, any debilitating chronic illness, or a calculated creatinine clearance of $\leq 50 \mathrm{~mL}$ minute. Weight, waist circumference, and blood pressure were measured after an overnight fast, and a blood sample was drawn. Plasma fasting glucose, serum total cholesterol, high-density lipoprotein (HDL) cholesterol, serum testosterone (T) levels and triglycerides were measured using enzymatic methods with an autoanalyzer. Metabolic syndrome was defined according to the criteria established in 2005 by the NCEP/ATP III. For the criteria for metabolic syndrome, abdominal obesity was defined as waist circumference $>102 \mathrm{~cm}$ in men and $>88 \mathrm{~cm}$ in women, according to the NCEP/ATP III obesity criteria. Metabolic syndrome was diagnosed in those who satisfied at least 3 of the following 5 criteria: waist circumference $>$ $102 \mathrm{~cm}$ in men, triglyceride concentration $>150 \mathrm{mg} / \mathrm{dL}$ or undergoing treatment for hypertriglyceridemia, HDL cholesterol concentration $<40$ $\mathrm{mg} / \mathrm{dL}$ in men or undergoing treatment for low HDL-C level, blood pressure > 130/85 $\mathrm{mm} \mathrm{Hg}$ or undergoing treatment for hypertension, and fasting plasma glucose level > $100 \mathrm{mg} / \mathrm{dL}$ or undergoing treatment for hyperglycemia (3). Serum testosterone levels were also evaluated and blood samples were taken between 08.00 and 10.00 in the fasting state. Analyses were completed using chisquare tests. Odds ratios (OR) were calculated. Statistical determinations were within the

\section{Table 3.}

$95 \%$ confidence interval (CI). All p values were two-tailed, and $p<0.05$ was considered statistically significant. The data were analyzed with an SPSSTM (SPSS version 13.0, Chicago, IL) statistical software package.

\section{Results}

Baseline demographic characteristics of the 513 participants are listed in Table 1. In the study population, 313 were patients with urolithiasis aged 24-67 years and 200 were patients without urolithiasis aged 31-64 years.

Serum T levels were detected in the lower limit $(<285$ $\mathrm{ng} / \mathrm{dL}$ ) on $112(35.7 \%)$ of the patients with stone disease and $32(16 \%)$ men with no stone disease. We have shown that lower $\mathrm{T}$ levels in men was associated with increased risk of urolithiasis (p 0.000, OR 2.93) (Table 2).

$161(51.4 \%)$ men in group 1 and 92 (46\%) men in group 2 were diagnosed with metabolic syndrome. In the MetS group, T level was found at lower limit $(<285 \mathrm{ng} / \mathrm{dl})$ more frequently in the ürolithiasis group (p 0.002, OR 2.71). Also in the group without MetS, $\mathrm{T}$ level was found at lower limit more frequently in the ürolithiasis group (p 0.001, OR 3.1). The prevalence of lower $T$ levels in men with

\section{Table 1.}

Demographic characteristics of the participants.

\begin{tabular}{|l|c|c|}
\hline $\mathbf{N}$ & $\begin{array}{c}\text { Stone (+) } \\
313\end{array}$ & $\begin{array}{c}\text { Stone }(-) \\
200\end{array}$ \\
\hline Age & $44.3 \pm 12.3$ & $42.7 \pm 13.3$ \\
\hline MetS $(+)$ & $161(51.4 \%)$ & $46(23 \%)$ \\
\hline MetS $(-)$ & $152(48.6 \%)$ & $154(77 \%)$ \\
\hline$T<285 \mathrm{ng} / \mathrm{dl}$ & $112(35.7 \%)$ & $32(16 \%)$ \\
\hline $\mathrm{T}>285 \mathrm{ng} / \mathrm{dl}$ & $201(64.3 \%)$ & $168(84 \%)$ \\
\hline MetS $(+)$ and T $<285 \mathrm{ng} / \mathrm{dl}$ & $64(20.4 \%)$ & $9(4.5 \%)$ \\
\hline MetS $(+)$ and T $>285 \mathrm{ng} / \mathrm{dl}$ & $97(30.9 \%)$ & $37(18.5 \%)$ \\
\hline MetS $(-)$ and T $<285 \mathrm{ng} / \mathrm{dl}$ & $48(15.3 \%)$ & $23(11.5 \%)$ \\
\hline MetS $(-)$ and $\mathrm{T}>285 \mathrm{ng} / \mathrm{dl}$ & $104(33.2 \%)$ & $131(65.5 \%)$ \\
\hline
\end{tabular}

\section{Table 2.}

The association between serum testosterone levels and urinary stone disease.

\begin{tabular}{|l|c|c|c|c|c|}
\hline STL & $\begin{array}{c}\text { Group 1 } \\
\text { (stone +) }\end{array}$ & $\begin{array}{c}\text { Group 2 } \\
\text { (stone -) }\end{array}$ & p & $\begin{array}{c}\text { Odds } \\
\text { ratio }\end{array}$ & Cl \\
\hline Overall & & & & & \\
$\mathrm{T}<285 \mathrm{ng} / \mathrm{dL}$ & $112(35.7 \%)$ & $32(16 \%)$ & 0.000 & 2.93 & $1.88-4.56$ \\
$\mathrm{~T} \geq 285 \mathrm{ng} / \mathrm{dL}$ & $201(64.3 \%)$ & $168(84 \%)$ & & & \\
\hline
\end{tabular}

Relation of urinary stone disease with metabolic syndrome and serum testosterone level.

\begin{tabular}{|l|c|c|c|c|c|}
\hline STL & Stone (+) & Stone $(-)$ & $\mathbf{p}$ & Odds ratio & CI \\
\hline For MetS (-) & $\mathrm{n}=152$ & $\mathrm{n}=154$ & & & \\
$\mathrm{~T}<285 \mathrm{ng} / \mathrm{dL}$ & $48(31.6 \%)$ & $23(14.9 \%)$ & 0.001 & 3.1 & $1.61-5.98$ \\
$\mathrm{~T} \geq 285 \mathrm{ng} / \mathrm{dL}$ & $104(68.4 \%)$ & $\begin{array}{c}131(85.1 \%) \\
\text { For MetS }(+)\end{array}$ & & & \\
$\mathrm{T}<285 \mathrm{ng} / \mathrm{dL}$ & $64(39,8 \%)$ & $9(19.6 \%)$ & 0.002 & 2.71 & $1.48-4.96$ \\
$\mathrm{~T} \geq 285 \mathrm{ng} / \mathrm{dL}$ & $97(60.2 \%)$ & $37(80.4 \%)$ & & & \\
\hline
\end{tabular}


Figure 1.

Relation of urinary stone disease with metabolic syndrome and serum testosterone level.

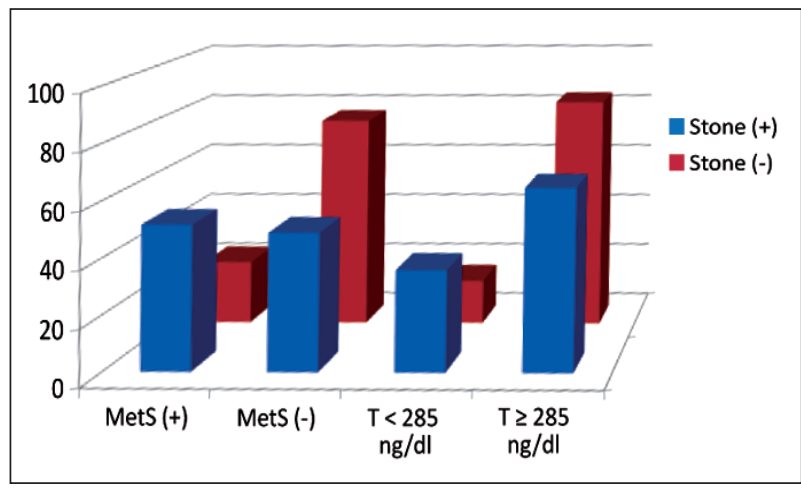

metabolic syndrome was higher than in men with nonMetS. The prevalance of the MetS in men with stone disease was higher than in men without stone disease (Table 3) (Figure 1)

\section{Discussion}

In our study, metabolic syndrome was associated with a significantly increased risk of urolithiasis and low testosterone level. A recent cross-sectional study from Italy demonstrated that individuals with MetS are twice as likely to have ultrasonographic evidence of nephrolithiasis. West and associates similarly showed that the odds of self-reported stone disease are approximately twice as likely in individuals with MetS than those without (25). There are a number of possible reasons for the association between MetS and nephrolithiasis. It has been demonstrated that features of MetS are associated with decreased urine $\mathrm{pH}$. One study noted that increasing insuline resistance (IR) (measured by comparing glucose disposal rates via euglycemic clamp with 24-hour urine studies) is associated with more acidic urine (26). Several investigators have determined that urine $\mathrm{pH}$ decreases with increasing BMI (27-30). They also found that the components of MetS significantly associated with urinary $\mathrm{pH}$ are BMI, serum glucose, and serum HDL (26). Low urine $\mathrm{pH}$ is a well-described feature of uric acid urolithiasis, so it is not surprising that people with features of MetS tend to preferentially have uric acid stones or risk factors for their development. A higher prevalence of type 2 diabetes (T2DM), glucose intolerance, and hypertriglyceridemia in pure uric acid stone formers has been reported (31). Similar to other studies, significant association was shown between the metabolic syndrome and urinary stone in our study.

At the same time, the metabolic syndrome is closely related to low testosterone levels. There is growing evidence that testosterone in men plays a major role not only in aspects of sexual health, but also in muscle and bone mass maintenance, erythropoiesis, and even glucose and lipid metabolism (32). Low testosterone levels are significantly associated with prevalence of MetS $(33,34)$. Men with prostate cancer ( $\mathrm{PCa}$ ) who undergo long-term androgen deprivation therapy (ADT) also have significantly higher rates of MetS than those who do not (35). Numerous stud- ies have demonstrated that MetS features such as HTN, obesity, hyperinsulinemia, T2DM, hyperglycemia, hypertriglyceridemia, as well as low HDL are associated with diminished serum testosterone levels (36-38). All these clinical sydromes or diseases are also associated with increased urinary stone prevalence. Our findings support the association of low $\mathrm{T}$ level and urolithiasis.

However, there are many studies that showing the relationship between high testosterone level and formation of urinary stone in the literature. In a study by Watson et al, a higher total serum testosterone level was found in stone-forming men than in non-stone forming controls (39). In literature there is no clinical study except this one regarding the relation between urolithiasis and serum $\mathrm{T}$ levels. But, in this study patient and control number are extremely lower. They have used total 55 patients. Of the 55 patients, 25 had no history of urolithiasis and 30 had a history of urolithiasis. In other words, totally 30 lithogenic patients have been evaluated to show the association of urolithiasis and serum $\mathrm{T}$ concentrations. Naghii et al. reported the role of androgenic sex steroids in a case study (40). It is not possible to explain this association with case studies. Further metabolic evaluations are needed to confirm this suggestion. We have more patients to demonstrate urolithiasis and serum $\mathrm{T}$ association. Our results also clearly show that serum $\mathrm{T}$ levels are lower in stone forming metabolic patients than non-stone forming patients with metabolic syndrome. Our findings also show the coexistence of low serum $\mathrm{T}$ levels and urolithiasis. Our previous and other works show the dicreased bone mineral density in stone formers $(41,42)$. Taking also into account that $\mathrm{T}$ has a significant role in bone mineralisation, our work supports the association of low serum $\mathrm{T}$ and urolithiasis.

Experimental studies in rats have demonstrated that testosterone promotes stone formation and that estrogen inhibits stone formation, although the mechanisms remain unclear (43). In contrast to these findings, we found low $\mathrm{T}$ levels in the patients with stone disease and higher prevalence of MetS in men with urolithiasis than in men without stone disease. Components of the metabolic syndrome such as obesity and HTN which have been shown in association with urolithiasis, can also be associated with low level of testosterone. In our opinion, levels of testosterone had no effect on stone formation, but the factors that cause stone formation affect level of testosterone. As a conclusion, urolithiasis is associated with low serum T levels, especially in patients with metabolic syndrome. Stone forming male patients should be evaluated for $\mathrm{T}$ deficiency and hypogonadism.

\section{References}

1. Gorbachinsky, I, Akpinar H, Assimos DG. Metabolic syndrome and urologic diseases. Rev Urol. 2010; 12:e157-80.

2. Eckel RH, Treating dyslipidemia of the metabolic syndrome: where's the evidence? Nat Clin Pract Endocrinol Metab. 2007; 3:437.

3. Huang PL, A comprehensive definition for metabolic syndrome. Dis Model Mech. 2009; 2:p. 231-7.

4. Binbay M1, Yuruk E, Akman Tet al. Updated epidemiologic study of urolithiasis in Turkey II: role of metabolic syndrome components on urolithiasis. Urol Res. 2012; 40:247-52. 
5. Stamatelou KK, et al. Time trends in reported prevalence of kidney stones in the United States: 1976-1994. Kidney Int. 2003; 63:1817-23.

6. Taylor EN, Stampfer MJ, Curhan GC. Obesity, weight gain, and the risk of kidney stones. JAMA. 2005; 293:455-62.

7. Taylor EN, Stampfer MJ, Curhan GC. Diabetes mellitus and the risk of nephrolithiasis. Kidney Int. 2005; 68:1230-5.

8. Curhan GC, et al. Body size and risk of kidney stones. J Am Soc Nephrol. 1998; 9:1645-52.

9. Jeong IG, et al. The association of metabolic syndrome and its components with serum prostate-specific antigen levels in a Koreanscreened population. Cancer Epidemiol Biomarkers Prev. 2010; 19:371-80.

10. Sakhaee K, Maalouf NM. Metabolic syndrome and uric acid nephrolithiasis. Semin Nephrol. 2008; 28:174-80.

11. Sakhaee K. Recent advances in the pathophysiology of nephrolithiasis. Kidney Int. 2009; 75:585-95.

12. Iba A. Insulin resistance increases the risk of urinary stone formation in a rat model of metabolic syndrome. BJU Int. 2010; 106:1550-4.

13. Yassin AA, Saad F, Gooren LJ. Metabolic syndrome, testosterone deficiency and erectile dysfunction never come alone. Andrologia. 2008; 40:259-264.

14. Kupelian V, Hayes FJ, Link CL, et al. Inverse association of testosterone and the metabolicsyndrome in men is consistent across race and ethnic groups. J Clin Endocrinol Metab. 2008; 93:3403-3410.

15. Robertson WG, Peacock $M$, Heyburn PJ, Hanes FA. Epidemiological risk factors in calcium stone disease. Scand J Urol Nephrol 1980; 53:15-30.

16. Soucie JM, Thun MJ, Coates RJ, et al. Demographic and geographic variability of kidney stones in the United States. Kidney Int. 1994; 46:893-899.

17. Fetter TR, Zimskind PD, Graham RH, Brodie DE. Statistical analysis of patients with ureteral calculi. JAMA 1963; 186:21-23.

18. Westbury EJ. Some observations on the quantitative analysis of over 1000 urinary calculi. Br J Urol. 1974; 46:215-227.

19. Lee YH, Huang WC, Tsai JY, et al. Epidemiological studies on the prevalence of upper urinary calculi in Taiwan. Urol Int. 2002; 68:172177.

20. Kohri K, Kodama M, Ishikawa Y, et al. Relationship betweenmetabolic acidosis and calcium phosphate urinary stone formation in women. Int Urol Nephrol. 1991; 23:307-316.

21. Marshall V, White RH, De Saintonage MC, et al. The natural history of renal and ureteral calculi. Br J Urol. 1975; 47:117-124.

22. Johnson CM, Wilson DM, O'Fallon WM, et al. Renal stone epidemiology: A 25-year study in Rochester, Minnesota. Kidney Int. 1979; 16:624-31.

23. Soucie JM, Thun MJ, Coates RJ, et al. Demographic and geographic variability of kidney stones in the United States. Kidney Int. 1994, 46:893-899.

24. Kaufman JM, Vermeulen A. The decline of androgen levels in elderly men and its clinical and therapeutic implications. Endocr Rev. 2005; 26:833-876.

25. West B, et al. Metabolic syndrome and self-reported history of kidney stones: the National Health and Nutrition Examination Survey (NHANES III) 1988-1994. Am J Kidney Dis. 2008. 51:741-7.

26. Maalouf NM, et al. Low urine $p H$ : a novel feature of the metabolic syndrome. Clin J Am Soc Nephrol. 2007; 883-8.

27. Li WM, et al. Association of body mass index and urine $\mathrm{pH}$ in patients with urolithiasis. Urol Res. 2009; 37:193-6.
28. Siener $R$, et al. The role of overweight and obesity in calcium oxalate stone formation. Obes Res. 2004; 12:106-13.

29. Taylor EN, Curhan GC. Body size and 24-hour urine composition. Am J Kidney Dis. 2006; 48:905-15.

30. Ekeruo WO et al. Metabolic risk factors and the impact of medical therapy on the management of nephrolithiasis in obese patients. J Urol 2004; 172: 159-63.

31. Sakhaee $K$, et al. Pathophysiologic basis for normouricosuric uric acid nephrolithiasis. Kidney Int. 2002; 62:971-9.

32. Yassin AA, Saad F, Gooren LJ. Metabolic syndrome, testosterone deficiency and erectile dysfunction never come alone. Andrologia. 2008; 40:259-64

33. Kupelian V. et al. Inverse association of testosterone and the metabolic syndrome in men is consistent across race and ethnic groups. J Clin Endocrinol Metab. 2008; 93:3403-10.

34. Goncharov NP, et al. Three definitions of metabolic syndrome applied to a sample of young obese men and their relation with plasma testosterone. Aging Male. 2008; 11:118-22.

35. Braga-Basaria $M$, et al. Metabolic syndrome in men with prostate cancer undergoing long-term androgen-deprivation therapy. J Clin Oncol. 2006; 24:3979-83.

36. Khaw KT, Barrett-Connor E. Blood pressure and endogenous testosterone in men: an inverse relationship. J Hypertens. 1988; 6:329-32.

37. Svartberg J, et al. Association of endogenous testosterone with blood pressure and left ventricular mass in men. The Tromso Study. Eur J Endocrinol. 2004; 150:65-71.

38. Phillips GB, Jing T, Heymsfield SB. Relationships in men of sex hormones, insulin, adiposity, and risk factors for myocardial infarction. Metabolism. 2003; 52:784-90.

39. Watson JM, Shrewsberry AB, Taghechian S, et al. Serum testosterone may be associated with calcium oxalate urolithogenesis. J Endourol. 2010; 24:1183-7.

40. Naghii MR, Hedayati M. Determinant role of gonadal sex hormones in the pathogenesis of urolithiasis in a male subject - a document for male predominancy (case study). Endocr Regul. 2010; 44:143-6.

41. Tugcu V, Ozbek E, Aras B, et al. Bone mineral density measurement in patients with recurrent normocalciuric calcium stone disease. Urol Res. 2007; 35:29-34.

42. Arrabal-Polo MA, Arrabal-Martin M, de Haro-Munoz T, et al. Mineral density and bone remodelling markers in patients with calcium lithiasis. BJU Int. 2011; 108:1903-8.

43. Lee YH, Huang WC, Chiang $H$, et al. Determinant role of testosterone in the pathogenesis of urolithiasis in rats. J Urol. 1992; 147:1134-1138.

\section{Correspondence}

Alper Otunctemur, MD (Corresponding Author) - alperotunctemur@yahoo.com Emin Ozbek, MD

Huseyin Besiroglu, MD

Okmeydani Training and Research Hospital, Department of Urology 34384 Sisli, Istanbul, Turkey

Suleyman Sami Cakir, MD

Bayburt State Hospital, Department of Urology, Bayburt, Turkey

Murat Dursun, MD

Bahcelievler State Hospital, Department of Urology, Istanbul, Turkey

Emre Can Polat, MD

Istanbul Medipol University, Faculty of Medicine, Department of Urology, Istanbul, Turkey

Levent Ozcan, MD

Derince Training and Research Hospital, Department of Urology, Kocaeli, Turkey 